\title{
Supervivencia de circuitos de técnicas de depuración extrarrenal continua en pacientes críticos con o sin anticoagulación convencional: estudio observacional prospectivo
}

\section{Circuit life span of continuous renal replacement therapy in critically ill patients with or without conventional anticoagulation: an observational prospective study}

doi.org/10.23938/ASSN.0008

M. Sanz ${ }^{1}$, F. Hidalgo' ${ }^{1}$, N. García-Fernández ${ }^{2}$

\section{RESUMEN}

Fundamento. El objetivo del presente estudio es describir la eficacia, seguridad y viabilidad, en pacientes críticos con técnica de depuración extrarrenal continua (TDEC) y diferente riesgo de hemorragia, de un sistema de anticoagulación convencional con perfusión continua de heparina no fraccionada (HNF) frente a no anticoagular usando lavados son suero fisiológico.

Material y métodos. Se trata de un estudio observacional prospectivo realizado en la Unidad de Cuidados Intensivos (UCI) desde octubre de 2013 hasta abril de 2016. Se incluyeron 61 pacientes que presentaron insuficiencia renal aguda (IRA) con requerimientos de TDEC y un total de 122 circuitos. Tanto los pacientes como los circuitos fueron divididos para su análisis en dos grupos: anticoagulados (AC) y no anticoagulados (No AC). La variable principal fue la supervivencia de los circuitos. Además se recogieron diferentes parámetros analíticos al comienzo del tratamiento y en el momento de coagulación del circuito.

Resultados. La distribución de pacientes anticoagulados y no anticoagulados fue similar. No se han encontrado diferencias significativas en la supervivencia de los circuitos entre ambos grupos ( 30,5 horas $\mathrm{AC}$ vs 34,9 horas No AC). Los pacientes con mayor morbilidad (trombopenia severa, coagulopatía, etc.) pertenecían al grupo que no recibió anticoagulación, sino lavados con suero fisiológico.

Conclusiones. En pacientes críticos con alto riesgo de sangrado las TDEC son viables sin anticoagulación más el empleo de lavados periódicos con suero fisiológico se comporta como una medida viable, segura y eficaz obteniendo una supervivencia de los circuitos similar a la de pacientes anticoagulados con HNF, evitando los riesgos y costes asociados a la anticoagulación.

Palabras clave: Anticoagulación. Pacientes críticos. Técnicas de depuración extrarrenal. Heparina. Solución salina.

\begin{abstract}
Background. The aim of this study was to describe the efficacy, security and viability of an anticoagulation system with continuous infusion of unfractionated heparin (UFH) versus one without any type of anticoagulant using $0.9 \%$ physiological saline washings, in critically ill patients with continuous renal replacement therapy (CRRT) and different risks of bleeding.
\end{abstract}

Methods. From October 2013 to April 2015 we conducted an observational prospective study in the intensive care unit (ICU). Sixty-one patients with acute kidney injury (AKI) and requiring CRRT were included, with 122 filters. Patients and filters were divided in two groups: anticoagulated (AC) and not anticoagulated (No AC). The main outcome measure was filter life span. Different analytical parameters were also collected at the beginning of treatment and at the moment of circuit coagulation

Results. The number of patients was similar in both groups. We did not find statistically significant differences between the two groups in filter life span (30.5 hours AC vs 34.9 hours No AC). Patients with increased morbidity (severe thrombocytopenia, coagulopathy, etc.) were included in the group that did not received anticoagulation but saline flushes.

Conclusions. CRRT without anticoagulation with saline flushes is a viable, safe and effective strategy in critically ill patients with high risk of bleeding. This approach achieves a circuit life span similar to that observed in anticoagulated patients with UFH; avoiding the risks and costs associated with anticoagulation.

Keywords: Anticoagulation. Critical patients. Extrarenal depuration techniques. Heparin. Saline solution.

\section{An. Sist. Sanit. Navar. 2017; 40 (1): 77-84}

1. Departamento de Anestesia y Cuidados intensivos. Clínica Universidad de Navarra. Pamplona. Navarra.

2. Departamento de Nefrología. Clínica Universidad de Navarra. Pamplona. Navarra.

Recepción: 30/09/2016

Aceptación provisional: 05/10/2016

Aceptación definitiva: 24/11/2016

\author{
Correspondencia: \\ María Sanz Ganuza \\ Departamento de Anestesia y Cuidados intensivos \\ Clínica Universidad de Navarra \\ Avda. Pío XII, 36 \\ 31008. Pamplona \\ Email: msganuza.1@alumni.unav.es
}




\section{INTRODUCCIÓN}

La insuficiencia renal aguda (IRA) es una complicación frecuente en las unidades de cuidados intensivos (UCI). Su incidencia ha aumentado en los últimos años y se asocia con un incremento de mortalidad y morbilidad ${ }^{1}$. La IRA se desarrolla entre un 5 y $25 \%$ de pacientes críticos según las series; de éstos, aproximadamente un $6 \%$ requirieren terapia de depuración extrarrenal (TDE) ${ }^{2,3}$.

En la actualidad, las terapias de depuración extrarrenal continuas (TDEC) son las más utilizadas en el paciente crítico, fundamentalmente porque permiten conseguir un adecuado balance hidroelectrolítico con mejor tolerancia hemodinámica ${ }^{4}$. Una de las complicaciones más frecuentes es la coagulación precoz del circuito. La frecuencia de estas interrupciones tiene implicaciones en la eficiencia del tratamiento. Esta complicación, además del impacto clínico sobre el paciente, supone un importante consumo de recursos y un alto coste de tratamiento. Adicionalmente, la coagulación repetida de los filtros conlleva pérdidas sanguíneas en el paciente, habitualmente anémico, y un consumo de factores de coagulación y de plaquetas ${ }^{5}$. Por todo ello, es esencial disponer de un sistema de anticoagulación, que garantice la supervivencia del circuito y reduzca al mínimo estos fenómenos.

Las guías más recientes recomiendan el citrato como anticoagulante de primera elección para las $\mathrm{TDEC}^{4}$. No obstante, la perfusión continua de heparina no fraccionada (HNF) sigue siendo el tratamiento anticoagulante más utilizado en la mayoría de las UCI debido al menor coste y/o su fácil monitorización ${ }^{6}$. Sin embargo, la anticoagulación sistémica presenta algunos riesgos asociados como mayor riesgo de hemorragia, consumo de plaquetas y factores de coagulación o fenómenos como la trombocitopenia inducida por heparina $(\mathrm{TIH})^{7}$. La anticoagulación con citrato también tiene inconvenientes como alteraciones metabólicas, especialmente en pacientes con disfunción hepática, y un coste más elevado ${ }^{8}$. Por otra parte, en un por- centaje elevado de pacientes críticos existe una contraindicación, absoluta o relativa, para ser anticoagulados por presentar un riesgo elevado de hemorragia debido a diferentes motivos (enfermedad hepática terminal, trombocitopenia, procedimientos quirúrgicos); o bien no se recomienda el empleo de citrato por presentar un riesgo elevado de asociarse a complicaciones. Por tanto, se trata de pacientes en los que utilizar los procedimientos habituales (HNF y/o citrato) puede causar complicaciones adicionales.

Una alternativa a cualquier método de anticoagulación es prescindir de la misma ${ }^{9}$, utilizando algún sistema que permita obviar por una parte, las complicaciones asociadas a los métodos de anticoagulación convencionales, y por otra, no suponga un obstáculo en la vida media de los circuitos que contraindique su empleo. En este sentido, se ha descrito la eficacia y viabilidad del uso intermitente de lavados con suero fisiológico ${ }^{10}$; método utilizado históricamente pero que carece de estudios controlados que confirmen los resultados iniciales en relación con su eficacia.

El objetivo de nuestro estudio es comparar la eficacia, seguridad y viabilidad, en pacientes críticos con diferente riesgo de hemorragia, de un sistema de anticoagulación convencional con perfusión continua de HNF versus no anticoagulación. Para ello valoramos diferentes parámetros relacionados con la supervivencia de los circuitos, así como la evolución de diferentes parámetros hematológicos, de coagulación y las necesidades transfusionales.

\section{MATERIAL Y MÉTODOS}

\section{Diseño del estudio}

Este trabajo fue diseñado como un estudio observacional prospectivo. Se realizó en la UCI de la Clínica Universidad de Navarra, que es una unidad con 12 camas que atiende a pacientes quirúrgicos, médicos y trasplantados (1.000 admisiones/año). El estudio fue aprobado por el Comité Ético de Investigación con medicamentos de Navarra. 
Todos los pacientes admitidos en la UCI desde octubre de 2013 hasta abril de 2015, con indicación de TDEC, fueron considerados candidatos para el estudio. Los criterios de inclusión fueron: pacientes entre 18 y 80 años de edad e IRA con requerimientos de TDEC. Los criterios de exclusión fueron: pacientes con sangrado activo, pacientes con historia de TIH y aquellos pacientes en los que los circuitos fueron cambiados de forma electiva a causa de procedimientos o pruebas intercurrentes.

Las indicaciones y la implementación de las TDEC se realizaron bajo la supervisión conjunta de anestesiólogos y nefrólogos. Se recogieron los datos de todos los circuitos que tuvieron que ser cambiados debido a la coagulación del filtro. La coagulación del filtro fue definida como la presencia de un coágulo visible en el circuito o una presión transmembrana mantenida mayor de $280 \mathrm{~mm} \mathrm{Hg}$, por lo que se produce la activación de la alarma de presión con riesgo de coagulación inmediata.

Tanto los pacientes del estudio como los circuitos analizados se dividieron para su análisis en dos grupos: anticoagulados (AC) y no anticoagulados (No AC).

\section{Variables de estudio}

Se analizaron las siguientes variables relacionadas con los pacientes: edad, simplified acute physiology score III (SAPS III), número de circuitos por paciente, duración del tratamiento con TDEC (días), indicación de TDEC (fallo renal secundario a sepsis, fallo hepático o cirugía extracorpórea y fallo renal primario), diámetro interno (French, Fr) y localización de la cánula de diálisis.

Además se recogieron las siguientes variables relacionadas con los circuitos: duración del circuito hasta su coagulación (horas), dosis de TDEC diaria y modalidad de TDEC: hemofiltración veno-venosa continua (HDFVVC) o hemodiálisis veno-venosa continua (HDVVC).

Por último se analizaron los requerimientos transfusionales durante el tratamiento (plasma fresco congelado, PFC, o concentra- do de hematíes, $\mathrm{CH}$ ) y los siguientes parámetros: hemoglobina, plaquetas, international normalized ratio (INR), tiempo de protrombina (TP), tiempo de tromboplastina parcial activado (TTPA), fibrinógeno y antitrombina (AT). Todos los parámetros mencionados fueron recogidos en dos momentos: al comienzo del tratamiento $\left(\mathrm{T}_{0}\right)$ y en el momento de coagulación del filtro $\left(\mathrm{T}_{1}\right)$.

\section{Protocolo de anticoagulación durante la TDEC}

Los pacientes recibieron anticoagulación sistémica con HNF. La dosis se ajustó en función del peso y del riesgo del paciente según el siguiente protocolo. Un bolo intravenoso de 2.000 UI seguido de una perfusión continua de $5-8 \mathrm{U} / \mathrm{Kg} / \mathrm{h}$ controlado mediante determinaciones de TTPA para mantener la razón entre 1,5-2, con ajustes cada 6 horas.

En pacientes con contraindicación para la anticoagulación, la técnica fue realizada sin ningún tipo de anticoagulación, con lavados de suero fisiológico al $0,9 \%$. Siguiendo nuestro protocolo habitual: cada hora durante las 2 primeras horas, y después cada 12 horas.

Los criterios que contraindicaban la anticoagulación fueron: plaquetas $<50.000 / \mathrm{L}$ y/o INR $>2$ y contraindicación para el uso de heparina (TIH, historia de alergia a la heparina...).

\section{Protocolo de TDEC}

Antes de iniciar la TDEC, se obtuvo el acceso vascular mediante la inserción de catéteres de doble luz en la vena yugular (13 o 13,5 Fr) o femoral (13,5 o 14 Fr) utilizando la técnica de Seldinger. El tratamiento fue realizado por multiFiltrate (Fresenius Medical Care) o Prisma-Flex (Hospal) en función de la disponibilidad de monitores; con un hemofiltro de membrana sintética de alto flujo, bien de polisulfona o AN69 según el monitor correspondiente.

Durante el periodo de recogida de datos y dependiendo de su situación clínica, 
todos los pacientes fueron tratados con dos tipos de modalidad de TDEC: HDFVVC o HDVVC.

El flujo sanguíneo prescrito habitualmente fue $200 \mathrm{ml} / \mathrm{min}$ o menor en función del catéter. El flujo de diálisis $\left(Q_{d}\right)$ y/o flujo de filtración $\left(Q_{u f}\right)$ fueron ajustados para garantizar una dosis de efluente de $30-35 \mathrm{ml} /$ $\mathrm{Kg} / \mathrm{h}$.

En la mayoría de los casos se utilizaron Dialisan (Hospal) como líquido de diálisis y Phoxilium (Hospal), como líquido de reposición en postdilución, durante la modalidad de filtración.

\section{Análisis estadístico}

Las variables continuas y categóricas se muestran como media y desviación estándar, y como número y porcentaje, respectivamente. Las características clínicas fueron comparadas entre ambos grupos (AC y No AC), usando el test exacto de Fisher, test de Mann-Withney o test $\chi^{2}$, según lo determinado por el tipo y la distribución de las variables.

Todos los análisis se llevaron a cabo con el programa Stata 14 (StataCorp. 2015.
Stata Statistical Software: Release 14. College Station, TX: StataCorp LP), con un nivel alfa bilateral de 0,05 .

\section{RESULTADOS}

Durante el periodo de estudio se incluyeron 61 pacientes tratados con TDEC. El análisis final se realizó en 50 pacientes por diferentes causas: 7 pacientes fueron excluidos al no lograr completar todos los datos y 4 pacientes porque murieron antes de la coagulación del circuito. Finalmente se incluyeron un total de 122 circuitos en el análisis de supervivencia mostrado en la figura 1.

En la tabla 1 se muestra los resultados de los datos clínicos y demográficos de los pacientes según recibieran o no anticoagulación. En nuestra muestra la distribución de pacientes anticoagulados con HNF y no anticoagulados fue similar: 21 pacientes recibieron anticoagulación en al menos un circuito, y 29 no fueron anticoagulados. Se encontraron diferencias significativas en la indicación de TDEC ( $\mathrm{p}=0,015)$ y en la necesidad de transfusión $(\mathrm{p}=0,004)$ específicamente de $\mathrm{CH}(\mathrm{p}=0,038)$.

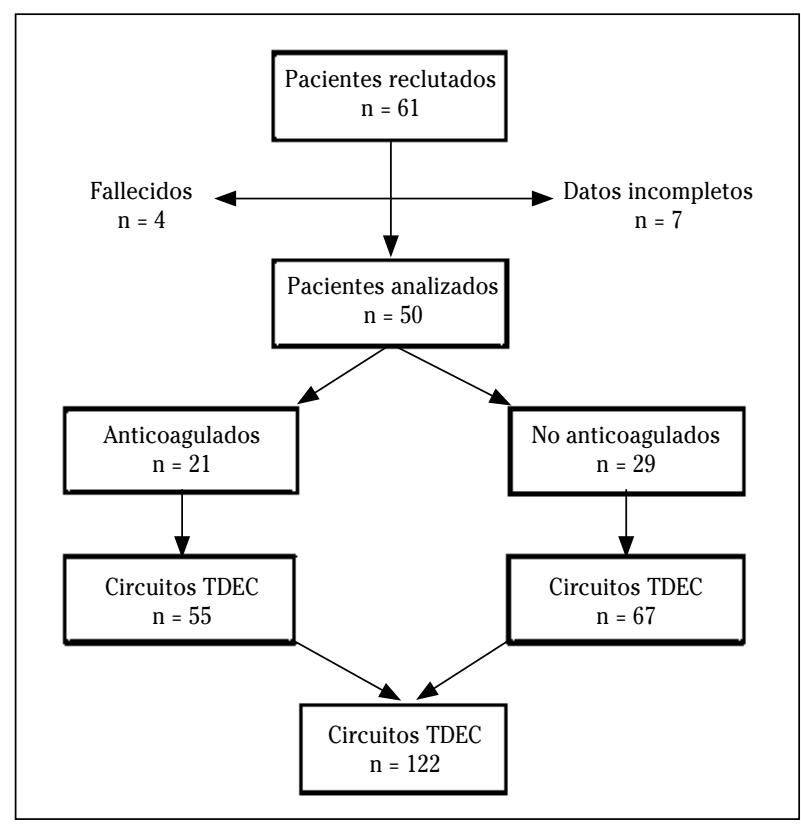

Figura 1.

Diagrama de selección de pacientes. 
Tabla 1. Resultado comparativo del análisis de pacientes con y sin anticoagulación

\begin{tabular}{|c|c|c|c|}
\hline & AC & No AC & $\mathbf{p}$ \\
\hline Pacientes (n) & 21 & 29 & \\
\hline Edad, media (DT) & $64(12,4)$ & $64(13,5)$ & 0,86 \\
\hline SAPS III & $59,1(19,2)$ & $61,3(16,9)$ & 0,67 \\
\hline № de circuitos por paciente & $4,6(3,2)$ & $4(4,1)$ & 0,24 \\
\hline Días de tratamiento & $7,2(6,2)$ & $8,7(10,8)$ & 0,87 \\
\hline \multicolumn{4}{|l|}{ Indicación de TDEC, n (\%) } \\
\hline IRA asociada a sepsis & $8(38,1)$ & $6(20,7)$ & \multirow{4}{*}{$\mathbf{0 , 0 1 5}$} \\
\hline IRA asociada a fallo hepático & $0(0)$ & $10(34,5)$ & \\
\hline IRA asociada a CEC & $5(23,8)$ & $5(17,2)$ & \\
\hline IRA primaria & $8(38,1)$ & $8(27,6)$ & \\
\hline Transfusión, n (\%) & $21(32,8)$ & $43(67,2)$ & 0,004 \\
\hline $\mathrm{CH}$ & $20(36,4)$ & $37(55,2)$ & $\mathbf{0 , 0 3 8}$ \\
\hline PFC & $1(1,8)$ & $11(16,4)$ & 0,084 \\
\hline
\end{tabular}

AC = anticoagulados; DT = desviación típica; SAPS III = Simplified Acute Physiology Score III; TDEC = técnica de depuración extrarrenal continua; IRA = insuficiencia renal aguda; CEC = circulación extracorpórea; $\mathrm{CH}=$ concentrado de hematíes; $\mathrm{PFC}=$ plasma fresco congelado

Las variables relacionadas con los circuitos se describen en la tabla 2. En 55 circuitos $(45,1 \%)$ se empleó la HNF y en 67 $(54,9 \%)$ se usaron lavados de suero fisiológico. No encontramos diferencias significativas ni en la supervivencia de los circuitos
(30,5 horas en AC versus 34,9 horas en No $\mathrm{AC})$, ni en la dosis prescrita entre ambos grupos (Fig. 2). Por el contrario sí observamos diferencias en la modalidad de TDEC $(\mathrm{p}=0,003)$.

Tabla 2. Resultados comparativos de circuitos analizados con o sin anticoagulación

\begin{tabular}{|c|c|c|c|}
\hline & AC & No $\mathrm{AC}$ & $\mathbf{p}$ \\
\hline Circuitos (n) & 55 & 67 & \\
\hline Vida media del filtro, horas & $30,5(21,7)$ & $34,9(23,1)$ & 0,26 \\
\hline Dosis, $\mathrm{ml} / \mathrm{Kg} / \mathrm{h}$ & $34,3(7.3)$ & $31,8(7,6)$ & 0,06 \\
\hline \multicolumn{4}{|l|}{ Modalidad de TDEC, n (\%) } \\
\hline Hemodiálisis & $25(34,2)$ & $48(65,8)$ & \multirow{2}{*}{$\mathbf{0 , 0 0 3}$} \\
\hline Hemofiltración & $30(61,2)$ & $19(38,8)$ & \\
\hline \multicolumn{4}{|c|}{ Diámetro del acceso vascular, n (\%) } \\
\hline$<13,5 \mathrm{Fr}$ & $11(52,4)$ & $19(65,5)$ & \multirow{2}{*}{0,35} \\
\hline$>13,5 \mathrm{Fr}$ & $10(47,6)$ & $10(34,5)$ & \\
\hline \multicolumn{4}{|c|}{ Localización del acceso vascular, n (\%) } \\
\hline Yugular & $18(85,7)$ & $23(79,3)$ & \multirow{2}{*}{0,72} \\
\hline Femoral & $3(14,3)$ & $6(20,7)$ & \\
\hline
\end{tabular}

$\mathrm{AC}=$ anticoagulados $; \mathrm{TDEC}=$ técnica de depuración extrarrenal continua $; \mathrm{Fr}=$ French 


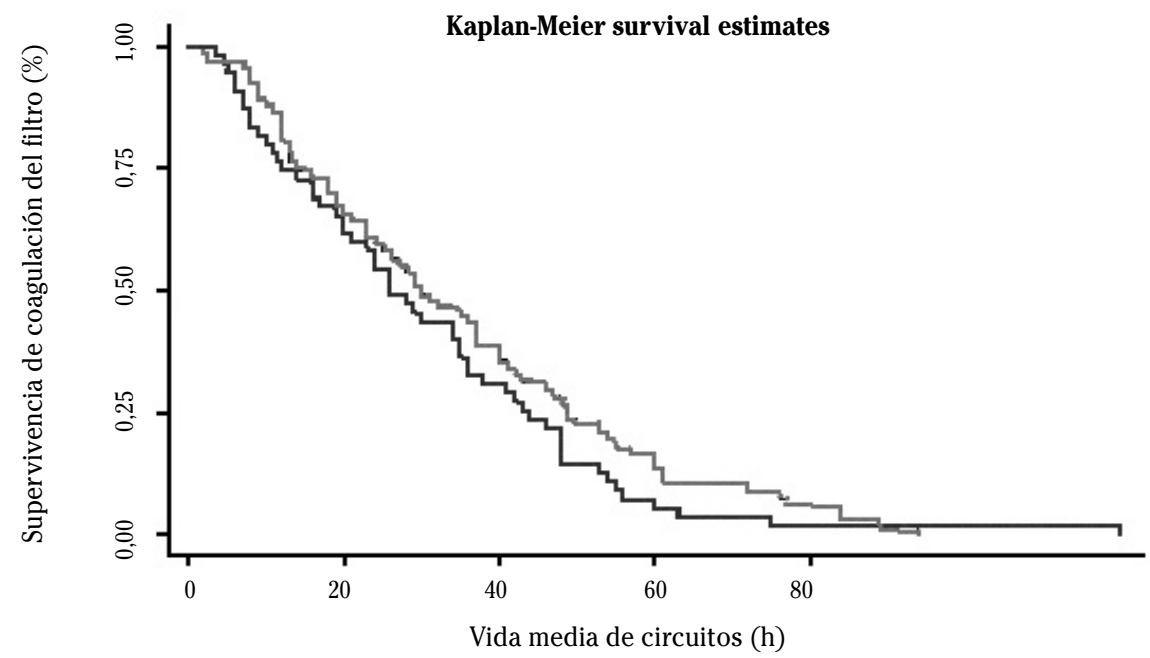

Number at risk

\begin{tabular}{llllll}
$\mathrm{AC}=\mathrm{No}$ & 67 & 45 & 26 & 11 & 4 \\
$\mathrm{AC}=\mathrm{HNF}$ & 55 & 36 & 17 & 4 & 1 \\
\cline { 4 - 4 } & & $\mathrm{AC}=\mathrm{No}$ & $\mathrm{AC}=\mathrm{HNF}$ \\
\hline
\end{tabular}

Figura 2. Gráfica de supervivencia de Kaplan-Meier de los circuitos de técnicas de depuración extrarrenal continuas según recibieran anticoagulación o no.

Tabla 3. Resultados comparativos de la evolución de parámetros analíticos con o sin anticoagulación.

\begin{tabular}{lccc}
\hline & AC & No AC & p \\
\hline $\begin{array}{l}\text { Basal (T) } \\
\mathrm{Hb}_{0}, \mathrm{~g} / \mathrm{L}\end{array}$ & $9,0(1,4)$ & $8,7(0,9)$ & 0,339 \\
\hline Plaquetas $_{0}, 10^{9} / \mathrm{L}$ & $131,3(72,7)$ & $78,2(68,0)$ & $<\mathbf{0 , 0 0 0 1}$ \\
\hline $\mathrm{TP}_{0}$, seg & $65,9(17,3)$ & $52,1(18,8)$ & $\mathbf{0 , 0 0 1}$ \\
\hline $\mathrm{INR}_{0}$ & $1,4(0,5)$ & $1,8(0,6)$ & $<\mathbf{0 , 0 0 1}$ \\
\hline $\mathrm{TPPA}_{0}$ & $1,5(0,6)$ & $4,1(21,8)$ & 0,687 \\
\hline Fibrinógeno $_{0}, \mathrm{mg} / \mathrm{dL}$ & $430,9(225,0)$ & $250,0(154,7)$ & $<\mathbf{0 , 0 0 0 1}$ \\
\hline $\mathrm{AT}_{0}$ & $72,4(21,4)$ & $50,6(24,7)$ & $<\mathbf{0 , 0 0 0 1}$ \\
\hline $\mathrm{Al}_{\text {coagularse }}\left(\mathrm{T}_{1}\right)$ & & & \\
$\mathrm{Hb}_{1}, \mathrm{~g} / \mathrm{L}$ & $8,8(1,2)$ & $8,6(0,9)$ & 0,278 \\
\hline Plaquetas $_{1}, 10^{9} / \mathrm{L}$ & $118,8(70,3)$ & $65,9(52,7)$ & $<\mathbf{0 , 0 0 0 1}$ \\
\hline $\mathrm{TP}_{1}$, seg & $68,1(22,3)$ & $54,6(20,4)$ & $\mathbf{0 , 0 0 0 6}$ \\
\hline $\mathrm{INR}_{1}$ & $1,5(0,7)$ & $1,7(0,6)$ & $\mathbf{0 , 0 0 0 3}$ \\
\hline $\mathrm{TTPA}_{1}$ & $1.5(0.7)$ & $1,4(0,4)$ & 0,286 \\
\hline Fibrinógeno $_{1}, \mathrm{mg} / \mathrm{dL}$ & $413,1(221,1)$ & $237,1(144,8)$ & $<\mathbf{0 , 0 0 0 1}$ \\
\hline $\mathrm{AT}_{1}$ & $72,8(20,8)$ & $53,5(27,3)$ & $<\mathbf{0 , 0 0 0 1}$ \\
\hline
\end{tabular}

$\mathrm{AC}=$ anticoagulados $; \mathrm{Hb}=$ hemoglobina; $\mathrm{TP}=$ Tiempo de protombina, $\mathrm{INR}=$ international normalized ratio; TTPA = tiempo de tromboplastina parcial activado; $\mathrm{AT}$ = antitrombina 
En la tabla 3 de resultados comparativos al inicio del tratamiento $\left(\mathrm{T}_{0}\right)$ y con la coagulación del circuito $\left(\mathrm{T}_{1}\right)$, en prácticamente todas las variables analizadas (plaquetas, TP, INR, fibrinógeno y AT) aparecen diferencias significativas $(\mathrm{p}<0,005)$.

\section{DISCUSIÓN}

En nuestro estudio hemos encontrado que en el paciente crítico, la supervivencia del circuito es similar entre los anticoagulados con heparina frente a los no anticoagulados. Así mismo, planteamos que esta ausencia de diferencia puede estar condicionada por la condición clínica del paciente y también porque en nuestro protocolo la no anticoagulación conlleva lavados con suero fisiológico.

Algunos estudios señalan una menor duración de las TDEC cuando se realizan sin anticoagulación en comparación con las que se utiliza algún método de anticoagulación ${ }^{11}$. Sin embargo, en nuestro estudio, al igual que otros trabajos ${ }^{12}$, encontramos que la supervivencia de los circuitos fue similar en ambos grupos (30,5 horas $\mathrm{AC}$ vs 34,9 horas No AC). Otro aspecto que confirma estos resultados, es el hecho de que ambos grupos consumieron el mismo número de circuitos por paciente (AC: 4,6 vs No AC: 4) en los mismos días de tratamiento (AC: 7,2 vs No AC: 8,7).

Esta similar vida media entre los circuitos sin anticoagulación y los anticoagulados puede explicarse por varias razones. Una de ellas es el empleo de lavados con suero fisiológico en el grupo de no anticoagulados, método que en algunos trabajos ${ }^{10}$ se asocia con una reducción de la coagulación de los filtros. También hay que tener en cuenta que todos los pacientes con disfunción hepática pertenecen al grupo que no recibió anticoagulación. Las características clínicas de estos pacientes (trombopenia, coagulopatía, mayor necesidad de transfusión...) influyen en la supervivencia de los filtros. Aunque, si comparamos la vida media de los circuitos en pacientes con hepatopatía (32,1 horas) frente el resto de pacientes ( 33,1 horas) obtenemos una vida media similar.
Por otra parte, en el grupo con anticoagulación predominaba la hemofiltración, modalidad que según algunos estudios ${ }^{13}$ puede contribuir a una menor duración de la vida media de los circuitos (Tabla 2). Y en este mismo sentido, en el grupo sin anticoagular la hemodiálisis fue mas indicada, implicando mas severidad del fracaso renal y mayor coagulopatía.

Los pacientes con mayor morbilidad (SAPS III elevado), en concordancia con otros trabajos, predominan en el grupo que no recibió anticoagulación ${ }^{14}$. Este grupo de pacientes representa el grupo de mayor riesgo de sangrado. Por ello en él se incluyen todos los pacientes con fallo hepático terminal con una alta incidencia de coagulopatía (trombopenia severa, INR prolongado, baja AT basal) corroborando datos previos ${ }^{9}$.

Por tanto, en este tipo de pacientes está plenamente justificado no utilizar ningún método de anticoagulación. Algunos trabajos recientes sugieren que el citrato podría incluso utilizarse en pacientes con fallo hepático, siempre y cuando se aumente la monitorización y se ajusten las dosis del mismo $^{15}$. Sin embargo, nuestros resultados demuestran que asociar a este grupo de pacientes de alto riesgo lavados periódicos con suero fisiológico es una alternativa segura y eficaz a cualquier tipo de anticoagulación.

Los pacientes no anticoagulados recibieron una cantidad significativamente mayor de hemoderivados (CH y PFC). Estos resultados aparentemente contradicen lo señalado por algunos autores ${ }^{10,12}$, que destacan una menor necesidad de transfusión con el método de lavados salinos comparado con la anticoagulación con HNF. Esta discordancia puede deberse a que, en nuestro estudio, los pacientes que no reciben anticoagulación son pacientes con patología más severa con mayor incidencia de anemia, trombopenia y coagulopatía, por lo cual precisan mayor soporte transfusional.

Nuestro estudio presenta algunas limitaciones. Se ha realizado en un único centro. En nuestra UCI existe una estrecha colaboración entre anestesiólogos y nefrólogos, lo cual probablemente no puede generalizarse a otros centros. El número de 
pacientes y de filtros es relativamente pequeño, por lo que probablemente algunos de nuestros hallazgos requerirían nuevos estudios para ser confirmados. Otra limitación es la falta de aleatorización de los grupos, ya que la asignación al grupo de heparina o al grupo de lavados dependía de la condición clínica del paciente, lo cual constituye un factor directamente relacionado con la supervivencia de los filtros.

Un aspecto destacable de nuestro estudio es el haber seleccionado un perfil de paciente que por sus circunstancias clínicas (hepatopatía terminal, coagulopatía, etc) puede beneficiarse de este manejo.

Las guías más recientes recomiendan en muchos pacientes la anticoagulación regional con citrato ${ }^{4}$. Sin embargo, existen numerosos pacientes no susceptibles de este tratamiento o centros donde no se ha implantado el mismo. Por tanto, pensamos que el mantener a los pacientes de alto riesgo hemorrágico sin anticoagulación, mediante lavados con suero fisiológico, es una alternativa viable, segura, eficaz y de aplicación práctica simple; con la que se evitan los riesgos y costes asociados a la anticoagulación.

\section{BIBLIOGRAFÍA}

1. Dennen P, Douglas IS, Anderson R. Acute kidney injury in the intensive care unit: an update and primer for the intensivist. Crit Care Med 2010; 38: 261-275.

2. Tolwani A. Continuous renal-replacement therapy for acute kidney injury. $\mathrm{N}$ Engl J Med 2012 27; 367: 2505-2514.

3. Uchino S, Kellum JA, Bellomo R, Doig GS, MoriMATSu H, Morgera $S$ et al. Acute renal failure in critically ill patients: a multinational, multicenter study. JAMA 2005 17; 294: 813-818.

4. Kidney Disease Outcomes Quality Initiative. KDIGO Clinical Practice Guidelines for Acute Kidney Injury. Kidney Int Suppl 2012; 2: 1-138.

5. JoAnnidis M, Oudemans-van StraAten HM. Clinical review: patency of the circuit in continuous renal replacement therapy. Crit Care 2007; 11: 218.

6. Uchino S, Bellomo R, Morimatsu H, Morgera S, SchetZ M, TAN I et al. Continuous renal replacement therapy: a worldwide practice survey. The beginning and ending supportive therapy for the kidney (B.E.S.T. kidney) investigators. Intensive Care Med 2007; 33: 1563-1570.

7. Oudemans-van Straten HM, Kellum JA, Bellomo R. Clinical review: anticoagulation for continuous renal replacement therapy-heparin or citrate? Crit Care 2011 24; 15: 202.

8. Stucker F, Ponte B, Tataw J, Martin PY, Wozniak H, Pugin J et al. Efficacy and safety of citratebased anticoagulation compared to heparin in patients with acute kidney injury requiring continuous renal replacement therapy: a randomized controlled trial. Crit Care 2015 18; 19: 91-015-0822-z.

9. Uchino S, Fealy N, Baldwin I, Morimatsu H, BelloMo R. Continuous venovenous hemofiltration without anticoagulation. ASAIO J 2004; 50: 76-80.

10. ZimbudZI E. Intermittent saline flushes or continuous saline infusion: what works better when heparin-free dialysis is recommended? Int J Nephrol Renovasc Dis 2013 15; 6: 65-69.

11. Karakala N, Tolwani A. We use heparin as the anticoagulant for CRRT. Semin Dial 2016; 29: 272-274.

12. Nagarik AP, Soni SS, Adikey GK, Raman A. Comparative study of anticoagulation versus saline flushes in continuous renal replacement therapy. Saudi J Kidney Dis Transpl 2010; 21 : 478-483.

13. Ricci Z, Ronco C, Bachetoni A, D'amico G, Rossi S, ALESSANDRI $\mathrm{E}$ et al. Solute removal during continuous renal replacement therapy in critically ill patients: convection versus diffusion. Crit Care 2006; 10: R67.

14. TAN HK, Baldwin I, Bellomo R. Continuous veno-venous hemofiltration without anticoagulation in high-risk patients. Intensive Care Med 2000; 26: 1652-1657.

15. Slowinski T, Morgera S, JoAnnidis M, Henneberg T, Stocker R, Helset E et al. Safety and efficacy of regional citrate anticoagulation in continuous venovenous hemodialysis in the presence of liver failure: the Liver Citrate Anticoagulation Threshold (L-CAT) observational study. Crit Care 2015 29; 19: 349-015-1066-7. 